\title{
Effect of inspiratory muscle training with load compared with sham training on blood pressure in individuals with hypertension: study protocol of a double- blind randomized clinical trial
}

Simone Regina Posser ${ }^{1}$, Carine Cristina Callegaro ${ }^{2}$, Marina Beltrami-Moreira ${ }^{1}$ and Leila Beltrami Moreira ${ }^{1,3^{*}}$

\begin{abstract}
Background: Hypertension is a complex chronic condition characterized by elevated arterial blood pressure. Management of hypertension includes non-pharmacologic strategies, which may include techniques that effectively reduce autonomic sympathetic activity. Respiratory exercises improve autonomic control over cardiovascular system and attenuate muscle metaboreflex. Because of these effects, respiratory exercises may be useful to lower blood pressure in subjects with hypertension.

Methods/design: This randomized, double-blind clinical trial will test the efficacy of inspiratory muscle training in reducing blood pressure in adults with essential hypertension. Subjects are randomly allocated to intervention or control groups. Intervention consists of inspiratory muscle training loaded with $40 \%$ of maximum inspiratory pressure, readjusted weekly. Control sham intervention consists of unloaded exercises. Systolic and diastolic blood pressures are co-primary endpoint measures assessed with $24 \mathrm{~h}$ ambulatory blood pressure monitoring. Secondary outcome measures include cardiovascular autonomic control, inspiratory muscle metaboreflex, cardiopulmonary capacity, and inspiratory muscle strength and endurance.
\end{abstract}

Discussion: Previously published work suggests that inspiratory muscle training reduces blood pressure in persons with hypertension, but the effectiveness of this intervention is yet to be established. We propose an adequately sized randomized clinical trial to test this hypothesis rigorously. If an effect is found, this study will allow for the investigation of putative mechanisms to mediate this effect, including autonomic cardiovascular control and metaboreflex.

Trial registration: ClinicalTrials.gov NCT02275377. Registered on 30 September 2014.

Keywords: Blood pressure, Clinical trial, Fitness, Hypertension, Physical therapy, Respiratory exercises, Sympathetic nervous system

\footnotetext{
* Correspondence: Ibmoreira@hcpa.edu.br

${ }^{1}$ Graduate Studies Program in Health Sciences: Cardiology and

Cardiovascular Sciences, Universidade Federal do Rio Grande do Sul (UFRGS),

Av. Jerônimo de Ornelas, 721, Porto Alegre, RS 90040-341, Brazil

${ }^{3}$ Hospital de Clínicas de Porto Alegre, Rua Ramiro Barcelos, 2350, room 943 ,

90035-903 Porto Alegre, RS, Brazil

Full list of author information is available at the end of the article
} 


\section{Background}

Essential hypertension is a multifactorial disease characterized by chronically elevated systolic blood pressure $(\geq 140 \mathrm{mmHg})$ or diastolic blood pressure $(\geq 90 \mathrm{mmHg})$ [1-4]. Hypertension is a major risk factor for targetorgan dysfunction, cardiovascular disease, and premature mortality $[3,4]$. The prevalence of hypertension in Brazil has been decreasing over the past three decades, but $30 \%$ of persons over 18 years of age still bear this condition [5].

The autonomic nervous system is a major determinant of systemic blood pressure and it might be a therapeutic target in hypertension $[6,7]$. Hyperactivity of the sympathetic nervous system plays a role in abnormal elevations of blood pressure [6-8]. Blood pressure variability is increased in hypertensive persons. This phenomenon has been linked to desensitization of baroreceptors, leading to an anomalous autonomic response [9, 10]. Betaadrenergic receptor blockers are an example of an effective pharmacologic approach to target the autonomic nervous system in hypertension [11].

Respiratory exercises are a non-pharmacologic intervention that might modulate autonomic nervous system activity and reduce blood pressure $[12,13]$. An exercise composed of controlled respiratory patterns with a slow respiratory rate has improved autonomic control and reduced blood pressure in hypertensive subjects [12]. Inspiratory muscle training - a different modality of respiratory exercise - was tested in one randomized clinical trial that included 13 participants [13]; in this study, inspiratory muscle training reduced 24 h systolic blood and diastolic blood pressure by 7.9 and $5.5 \mathrm{mmHg}$, respectively, and also improved autonomic cardiovascular control. Respiratory muscle training might also have reduced sympathetic activity through attenuation of muscle metaboreflex $[14,15]$.

\section{Methods/design}

\section{Study aim}

This study aims to test the efficacy of inspiratory muscle training in reducing blood pressure in subjects with essential hypertension. It will further investigate the effects of this intervention on inspiratory metaboreflex - a putative mechanism for blood pressure reduction. The conceptual hypothesis is that inspiratory muscle training reduces mean $24 \mathrm{~h}$ blood pressure through modulation of sympathetic nervous system activity and muscle metaboreflex.

\section{Study design}

This is a double-blind, parallel-group, sham-controlled randomized clinical trial. Participants are randomly assigned to receive inspiratory muscle training with a load equivalent to $40 \%$ of maximum inspiratory pressure or inspiratory muscle training sham (no load) for 8 weeks. The study flowchart is depicted in Fig. 1. Participants are followed on weekly visits. Study outcomes are assessed at the end of the eighth week. The study timeline is summarized in Additional file 1: Figure S1, where detailed

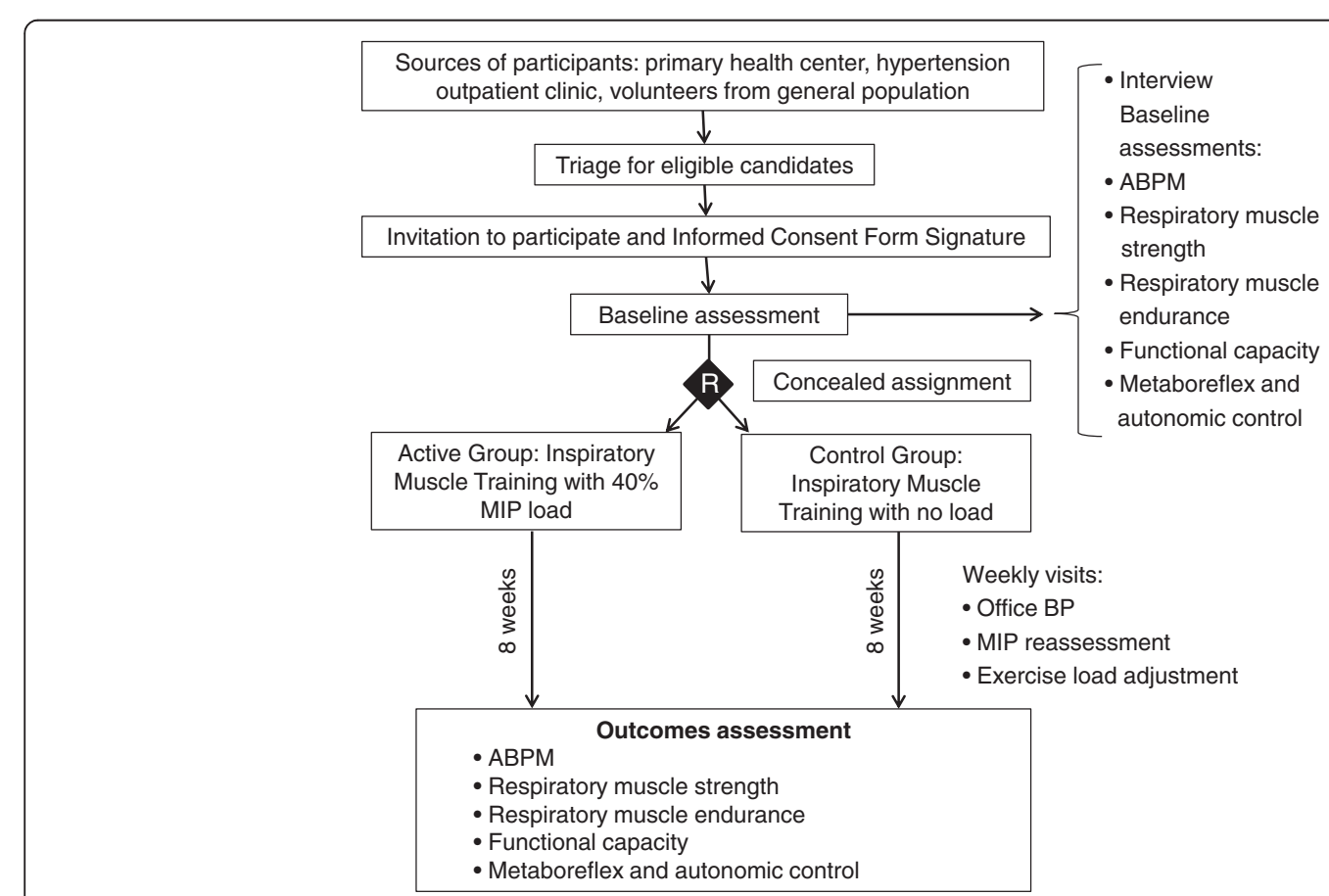

Fig. 1 Study flow diagram. BP blood pressure, ABPM ambulatory blood pressure monitoring, MIP maximum inspiratory pressure; $R$ randomization 
information on methods of assessment and protocols can be found.

\section{Study context}

Participants will be recruited from patients receiving follow-up care at the hypertension clinic or the primary health care center at the Hospital de Clínicas de Porto Alegre, a tertiary, university-affiliated teaching hospital. Public announcements (radio, newspapers, and website) will also call for volunteers among residents of the city of Porto Alegre.

\section{Eligibility criteria}

The study includes both male and female subjects, aged $\geq 35$ and $<65$ years, previously diagnosed with essential hypertension, with a systolic blood pressure $\geq 140 \mathrm{mmHg}$ or diastolic blood pressure $\geq 90 \mathrm{mmHg}$. Subjects receiving no pharmacologic therapy or taking only a thiazide diuretic are eligible to enter the study. Participants must have body mass index $<30 \mathrm{~kg} / \mathrm{m}^{2}$ [16], practice $<150 \mathrm{~min}$ of moderate or intense physical activity per week, according to the International Physical Activity Questionnaire [17, 18], and have inspiratory muscle strength $\geq 70 \%$ of predicted [19].

Participants are excluded from the study if they have: office blood pressure $\geq 160 / 100 \mathrm{mmHg}$; severe dyspnea; diabetes mellitus; orthopedic, musculoskeletal, or neurologic limitations; significant cognitive impairment; a current or past history of deep venous thrombosis; a history of myocardial infarction or stroke in the previous 6 months; congestive heart failure; unstable angina; or pulmonary disease of any etiology (including asthma and chronic obstructive pulmonary disease); or if they are active smokers. Women who are pregnant or breastfeeding are excluded from this study. Subjects are not eligible if they participated in another clinical trial in the previous 30 days, or if there is any other condition that might impair their ability to participate in the study or follow the protocol, at the discretion of the recruiter.

Participants are also directed not to change pharmacologic or non-pharmacologic treatment for hypertension (including changes in prescription or changes in the of level physical activity) during their participation in this study.

\section{Study outcomes}

The co-primary outcomes measures are changes from baseline to end of study in mean $24 \mathrm{~h}$ systolic and diastolic blood pressure, as evaluated by ambulatory blood pressure monitoring.

Secondary outcome measures are changes from baseline to end of study in mean daytime systolic and diastolic blood pressure; changes from baseline to end of study in mean nighttime systolic and diastolic blood pressure; heart rate variability; systolic blood pressure variability; aerobic capacity; inspiratory metaboreflex; and autonomic control.

\section{Randomization, allocation, blinding, and confidentiality}

A randomization sequence has been generated using Randomization.org in blocks of four to either group 1 - inspiratory muscle training with $40 \%$ of maximal inspiratory pressure - or group 2 - sham inspiratory muscle training. An independent person not involved in this study possesses the computer-generated randomization sequence. A research assistant not involved in participant assessment obtains the code and sets the load in each device, according to randomization. A black tape hides the loading spring from the participant and from the investigators assessing outcomes.

There are no foreseen circumstances when un-blinding is permissible. When data collection is completed, the person responsible for the randomization list will provide assignment information to the investigators. Patient's forms and other data sources are kept in a locked cabinet. Data are anonymized when entered into the database to protect confidentiality. Only investigators formally associated with the project will have access to the final study database.

\section{Studied variables and methods of assessment}

Participants are assessed as described in Additional file 1. Briefly, baseline physical activity is quantified using the International Physical Activity Questionnaire long form [20]. Body mass index is calculated as mass $(\mathrm{kg})$ divided by the square of the height $(\mathrm{m})$ and categorized as recommended by the World Health Organization [21]. Ambulatory blood pressure monitoring is performed with Spacelabs 90207 devices (Redmond, WA, USA). The protocol includes blood pressure measurement every 15 min during the daytime (6 a.m. to 10 p.m.) and every 20 min during the nighttime (10 p.m. to 6 a.m.) [22]. Functional capacity is assessed during exercise through $\mathrm{O}_{2}$ and $\mathrm{CO}_{2}$ expiration fraction in a metabolic system. Maximum oxygen consumption in each breath is recorded in $\mathrm{ml} /(\mathrm{Kg} / \mathrm{min})[23,24]$.

Maximum inspiratory and maximum expiratory pressure serve as indicators of inspiratory and expiratory muscle strength, respectively. Maximum inspiratory and maximum expiratory pressure are assessed using a digital pressure manometer (MVD-300, Microhard System, Globalmed, Porto Alegre, Brazil) [25, 26].

Inspiratory muscle endurance is determined using the incremental test proposed by Martyn et al. [27] using the Powerbreathe Plus system (London, UK). The test is stopped when the subject cannot open the inspiratory valve or desires to stop the test owing to respiratory exhaustion (quantified using the modified Borg scale). 
Blood pressure variability, heart rate variability, and the cardiac vagal tone are assessed to evaluate autonomic cardiovascular control. Non-invasive continuous blood pressure curves, heart rate, and electrocardiogram tracing are obtained simultaneously at a frequency of $1000 \mathrm{~Hz}$ using a Biopac MP150 (Biopac, California, EUA). Data are registered in a computer with biologic signal conversion capabilities.

The muscle metaboreflex is the adaptive blood flow redistribution from the peripheral circulation into the vascular bed of metabolically active (exercised) muscles [28]. The metaboreflex intensity is inversely related to one's fitness. Inspiratory muscle metaboreflex is assessed by causing diaphragmatic fatigue and detecting peripheral blood flow reduction [28, 29]. Monitored parameters include: oxygen saturation, carbon dioxide partial expiratory tension, mean arterial pressure, and venous occlusion plethysmography. A detailed protocol can be found in Additional file 1.

\section{Intervention}

Participants are extensively instructed on how to perform home-based inspiratory muscle training assisted by a Power Breathe Plus (London, UK) device. For participants' safety, research staff are responsible for device hygiene before and after the intervention period. To perform the exercise training, subjects should be seated, obstruct the nose with a nasal clip, and breathe in and out through the mouthpiece. The goal is to keep diaphragmatic breathing at $10-15$ respirations per minute for a total of $30 \mathrm{~min}$ per day, 7 days a week.

Participants receiving active inspiratory muscle training (intervention group) perform the exercises with a load equivalent to $40 \%$ of maximal inspiratory pressure. Those receiving the sham intervention (the control group) follow the same instructions but have no load applied to the respiratory exercises. The sham intervention was chosen as comparator because it is the most similar intervention available. It allows for blinding of investigators and participants, and is not expected to reproduce the effects of loaded exercises.

The complete study protocol consists of 56 daily training sessions completed over 8 weeks. During the scheduled weekly visit to the study center, participants are questioned about possible discomforts associated with the intervention, which may include: headaches, nausea, intense muscle fatigue, muscle cramps, and chest pain. Respiratory muscle strength is then reassessed; inspiratory threshold loading is adjusted accordingly, keeping a $40 \%$ maximum inspiratory load for the intervention group throughout the study. The staff member who receives information about treatment allocation and adjusts the load neither participates in any assessments nor communicates with participants.
Participants in both groups receive a journal to register date, time, duration of training session, and possible symptoms or discomforts associated to the intervention. This serves as a mean to stimulate and estimate adherence to intervention. Adherence to the protocol is also reinforced on weekly follow-up phone calls.

\section{Sample size calculation}

Sample size was estimated based on a small randomized clinical trial on the effect of inspiratory muscle training on blood pressure of hypertensive subjects in Brazil [13]. That study found an $8 \mathrm{mmHg}$ difference in systolic blood pressure between the active treatment and the control group (standard deviation, $10 \mathrm{mmHg}$ ). To detect this difference with power of $80 \%$, and assuming an alpha error of $5 \%$, the study should include 26 participants per group. We opted for a $20 \%$ inflation of this number to account for loss of follow-up and consent withdrawal - therefore, a total of 60 participants will be included and assigned to either intervention or sham treatment.

\section{Data management and statistical analysis plan}

Data recorded in printed forms are kept in locked cabinets. Information is entered on Microsoft Excel spreadsheets by one person and reviewed by another. Numeric continuous variables, including blood pressure in the primary outcome measures, will be described as mean \pm standard deviation or as median (interquartile range). Comparison between groups will be performed using Student's $t$ test or the Mann-Whitney U test, depending on variable distribution. As a superiority trial, differences in autonomic cardiovascular control and metaboreflex between groups will be tested with two-sided repeatedmeasures ANOVA. Results will be analyzed as per intention-to-treat. Participants who drop out and do not complete outcome assessment will be compared with those who completed the protocol but their data will be excluded from analysis. Statistical significance will be considered for $P<0.05$. All analyses will be performed using PASW Statistics $18^{\circ}$ (International Business Machines Corp., New York, NY).

\section{Results communication}

A summary of the main findings in non-technical language will be sent to participants after study completion. Scientific papers written according to the CONSORT recommendations will be submitted for peer-reviewed publication. Study results will also be communicated as abstracts and presentations in local, national, and international scientific meetings. 


\section{Discussion}

The effect of inspiratory muscle training on blood pressure in hypertensive individuals is not yet established. A significant reduction in blood pressure was observed in a small randomized clinical trial with six and five subject allocated to intervention and control groups, respectively [13]. Although these findings do not provide enough evidence to support the efficacy of inspiratory muscle training in blood pressure control, they are encouraging. We propose an adequately sized randomized clinical trial to test the hypothesis that inspiratory muscle training reduces blood pressure in persons with hypertension. If the hypothesis is confirmed, this study will also allow for investigation of putative mechanisms mediating such an effect. We will assess the effects of inspiratory muscle training on autonomic cardiovascular control and metaboreflex in subjects with hypertension - data that, to the best of our knowledge, are lacking in the medical literature.

The evaluation of inspiratory muscle metaboreflex is one of the strengths of this study. Inspiratory muscle training attenuated the metaboreflex in healthy subjects [30] and in persons with chronic heart failure [31]. In healthy subjects, decreased inspiratory work through assisted ventilation increases exercise time on a cycle ergometer by $14 \%$ [32]; a similar intervention has been shown to attenuate quadriceps fatigue during exercise [9]. This effect is probably due to inhibition of inspiratory muscle metaboreflex [10].

The effects of inspiratory muscle training deserve to be better understood given the potential to help control blood pressure in hypertensive patients. The results of this study will provide evidence on the effects of inspiratory muscle training on blood pressure, in addition to inspiratory muscle metaboreflex, autonomic cardiovascular control, and other physiologic and metabolic aspects. These data will provide mechanistic insights, as well as useful clinical information for the management of hypertension.

\section{Trial status}

The trial is enrolling participants.

\section{Additional file}

Additional file 1: Supplementary material. Methods for assessment of study outcomes and other variables. Figure S1. Schedule of enrollment, intervention, and assessments. Figure S2. Inspiratory muscle

metaboreflex induction protocol flowchart. (DOCX 362 kb)

\section{Abbreviations}

ANOVA, analysis of variance; CONSORT, Consolidated Standards of Reporting Trials

\section{Funding}

This project is funded by the Fundo de Incentivo a Pesquisa (FIPE), at Hospital de Clínicas de Porto Alegre. The funding agency had no role in study design and implementation, and will have no role in data analysis and interpretation, or the decision to publish trial results.

\section{Authors' contributions}

SRP projected the trial protocol, drafted this manuscript, collected data, and will perform data analysis and interpretation. MBM offered suggestions on trial design and data analysis, collaborated in the writing and critical review of this manuscript, and will collaborate in data interpretation. LBM and CCC contributed to protocol development and writing of this manuscript; they will perform data analysis and interpretation, critical revision and final approval of future research communications. All authors read and approved the final version of this manuscript.

\section{Authors' information}

SRP, PT, MSc is a PhD student in the Graduate Studies Program in Health Sciences: Cardiology and Cardiovascular Sciences, Universidade Federal do Rio Grande do Sul, Porto Alegre, RS.

CCC, PT, PhD is Professor of Physiotherapy at University of Cruz Alta, Cruz Alta, RS

MBM, MD, PhD collaborates as a volunteer visiting scholar in this project. LBM MD, PhD is a cardiologist and Professor of Clinical Pharmacology at Universidade Federal do Rio Grande do Sul, Porto Alegre, RS, Brazil.

\section{Competing interests}

The authors declare that they have no competing interests.

\section{Ethics approval and consent to participate}

Participants will receive a thorough oral and written description of the study goals, intervention, potential risks, and benefits involved, as well as efforts put in place to protect personal and health information. Participants have the opportunity to ask questions, which are answered by the investigator. After information, a consent form is offered for the participant to sign (in Portuguese only, available upon request). It is clarified that participants can withdraw the consent and quit the study at any time. If any medical attention is needed while participants are performing assessments in the research center, immediate medical care will be provided at the Hospital de Clínicas de Porto Alegre. The study protocol and its informed consent form have been approved by the Institutional Review Board of Hospital de Clinical de Porto Alegre (protocol number 140405) and is registered at ClinicalTrials.gov (NCT2275377). Any protocol amendments are subjected to institutional review board approval and will later be published at

ClinicalTrials.gov.

\section{Author details}

${ }^{1}$ Graduate Studies Program in Health Sciences: Cardiology and Cardiovascular Sciences, Universidade Federal do Rio Grande do Sul (UFRGS), Av. Jerônimo de Ornelas, 721, Porto Alegre, RS 90040-341, Brazil. 'Graduate Program in Integral Attention to Health (PPGAIS- UNICRUZ/UNIJUI). Universidade de Cruz Alta, Rodovia Municipal Jacob Della Méa, Km 5.6, Cruz Alta, RS 98020-290, Brazil. ${ }^{3}$ Hospital de Clínicas de Porto Alegre, Rua Ramiro Barcelos, 2350, room 943, 90035-903 Porto Alegre, RS, Brazil.

Received: 11 December 2015 Accepted: 20 July 2016

Published online: 02 August 2016

References

1. Mancia G, Fagard R, Narkiewicz K, Redon J, Zanchetti A, Bohm M, et al. 2013 ESH/ESC guidelines for the management of arterial hypertension: the Task Force for the Management of Arterial Hypertension of the European Society of Hypertension (ESH) and of the European Society of Cardiology (ESC). Eur Heart J. 2013:34(28):2159-219.

2. Nobre F. Introduction: Brazilian guidelines on hypertension VI. Brazilian Society of Cardiology, Brazilian Society of Hypertension, Brazilian Society of Nephrology. J Bras Nefrol. 2010;32 Suppl 1:iii.

3. Williams B, Poulter NR, Brown MJ, Davis M, Mclnnes GT, Potter JF, et al. Guidelines for management of hypertension: report of the fourth working party of the British Hypertension Society, 2004-BHS IV. J Hum Hypertens. 2004;18(3):139-85. 
4. ESH/ESC Task Force for the Management of Arterial Hypertension. 2013 Practice guidelines for the management of arterial hypertension of the European Society of Hypertension (ESH) and the European Society of Cardiology (ESC). ESH/ESC Task Force for the Management of Arterial Hypertension. J Hypertens. 2013;31(10):1925-38.

5. Picon RV, Fuchs FD, Moreira LB, Riegel G, Fuchs SC. Trends in prevalence of hypertension in Brazil: a systematic review with meta-analysis. PLoS One. 2012;7(10):e48255

6. Callegaro CC, Moraes RS, Negrao CE, Trombetta IC, Rondon MU, Teixeira MS, et al. Acute water ingestion increases arterial blood pressure in hypertensive and normotensive subjects. J Hum Hypertens. 2007;21(7):564-70.

7. Rondon MU, Laterza MC, de Matos LD, Trombetta IC, Braga AM, Roveda F, et al. Abnormal muscle metaboreflex control of sympathetic activity in never-treated hypertensive subjects. Am J Hypertens. 2006;19(9):951-7.

8. Guzzetti S, Piccaluga E, Casati R, Cerutti S, Lombardi F, Pagani M, et al. Sympathetic predominance in essential hypertension: a study employing spectral analysis of heart rate variability. J Hypertens. 1988:6:711-7.

9. Romer LM, Lovering AT, Haverkamp HC, Pegelow DF, Dempsey JA. Effect of inspiratory muscle work on peripheral fatique of locomotor muscles in healthy humans. J Physiol. 2006;571(Pt 2):425-39.

10. Ribeiro JP, Chiappa GR, Callegaro CC. The contribution of inspiratory muscles function to exercise limitation in heart failure: pathophysiological mechanisms. Rev Bras Fisioter. 2012;16(4):261-7.

11. Wiysonge CS, Bradley HA, Volmink J, Mayosi BM, Mbewu A, Opie LH. Betablockers for hypertension. Cochrane Database Syst Rev. 2012;11:CD002003. doi:10.1002/14651858.CD002003.pub4.

12. Hunt BE, Fahy L, Farquhar WB, Taylor JA. Quantification of mechanical and neural components of vagal baroreflex in humans. Hypertension. 2001;37(6):1362-8.

13. Ferreira JB, Plentz RDM, Stein C, Casali KR, Arena R, Lago PD. Inspiratory muscle training reduces blood pressure and sympathetic activity in hypertensive patients: a randomized controlled trial. Int J Cardiol. 2013;166(1):61-7.

14. Witt JD, Guenette JA, Rupert JL, McKenzie DC, Sheel AW. Inspiratory muscle training attenuates the human respiratory muscle metaboreflex. J Physiol. 2007:584(Pt 3):1019-28.

15. McConnell AK, Lomax M. The influence of inspiratory muscle work history and specific inspiratory muscle training upon human limb muscle fatigue. J Physiol. 2006;577(Pt 1):445-57.

16. Jensen MD, Ryan DH, Donato KA, Apovian CM, Ard JD, Comuzzie AG, et al. Executive summary: guidelines (2013) for the management of overweight and obesity in adults: A Report of the American College of Cardiology/ American Heart Association Task Force on Practice Guidelines and The Obesity Society Published by The Obesity Society and American College of Cardiology/ American Heart Association Task Force on Practice Guidelines. Based on a systematic review from the The Obesity Expert Panel, 2013. Obesity (Silver Spring). 2014;22 Suppl 2:S5-39.

17. Hallal PC, Victora CG, Wells JC, Lima RC. Physical inactivity: prevalence and associated variables in Brazilian adults. Med Sci Sports Exerc. 2003;35(11):1894-900.

18. Hallal PC, Simoes E, Reichert FF, Azevedo MR, Ramos LR, Pratt M, et al. Validity and reliability of the telephone-administered international physical activity questionnaire in Brazil. J Phys Act Health. 2010;7(3):402-9.

19. Neder JA, Andreoni S, Lerario MC, Nery LE. Reference values for lung function tests. II. Maximal respiratory pressures and voluntary ventilation. Braz J Med Biol Res. 1999;32(6):719-27.

20. Adams MA, Ding D, Sallis JF, Bowles HR, Ainsworth BE, Bergman P, et al. Patterns of neighborhood environment attributes related to physical activity across 11 countries: a latent class analysis. Int J Behav Nutr Phys Act. 2013;10:34

21. World Health Organization. Body Mass Index - BMI. http://www.euro.who. int/en/health-topics/disease-prevention/nutrition/a-healthy-lifestyle/bodymassindex-bmi (2016). Accessed 19 May 2016

22. Grupo de Trabalho MAPA, Grupo de Trabalho MRPA. V Brazilian quidelines for ambulatory monitoring of arterial pressure and III Brazilian guidelines for home monitoring of blood pressure]. J Bras Nefrol. 2011;33(3):365-88.

23. American Thoracic Society, American College of Chest Physicians. ATS/ACCP Statement on cardiopulmonary exercise testing. Am J Respir Crit Care Med. 2003;167(2):211-77.

24. Winkelmann ER, Chiappa GR, Lima CO, Viecili PR, Stein R, Ribeiro JP. Addition of inspiratory muscle training to aerobic training improves cardiorespiratory responses to exercise in patients with heart failure and inspiratory muscle weakness. Am Heart J. 2009;158(5):768. e1-7.

25. Sobush DC, Dunning 3rd M. Assessing maximal static ventilatory muscle pressures using the 'bugle' dynamometer. Suggestion from the field. Phys Ther. 1984;64(11):1689-90.

26. Callegaro CC, Taylor JA. Age-related effects of vagotonic atropine on cardiovagal baroreflex gain. Neurobiol Aging. 2012;33(2):368-74.

27. Martyn JB, Moreno RH, Pare PD, Pardy RL. Measurement of inspiratory muscle performance with incremental threshold loading. Am Rev Respir Dis. 1987;135(4):919-23.

28. Dempsey JA, Romer L, Rodman J, Miller J, Smith C. Consequences of exercise-induced respiratory muscle work. Respir Physiol Neurobiol. 2006; 151(2-3):242-50

29. St Croix CM, Morgan BJ, Wetter TJ, Dempsey JA. Fatiguing inspiratory muscle work causes reflex sympathetic activation in humans. J Physiol. 2000;529(Pt 2):493-504.

30. Callegaro CC, Ribeiro JP, Tan CO, Taylor JA. Attenuated inspiratory muscle metaboreflex in endurance-trained individuals. Resp Physiol Neuro. 2011;177:24-9.

31. Chiappa GR, Roseguini BT, Vieira PJ, Alves CN, Tavares A, Winkelmann ER, Ferlin EL, Stein R, Ribeiro JP. Inspiratory muscle training improves blood flow to resting and exercising limbs in patients with chronic heart failure. J Am Coll Cardiol. 2008;51:1663-71.

32. Harms CA, Wetter TJ, St Croix CM, Pegelow DF, Dempsey JA. Effects of respiratory muscle work on exercise performance. J Appl Physiol (1985). 2000:89(1):131-8.

\section{Submit your next manuscript to BioMed Central and we will help you at every step:}

- We accept pre-submission inquiries

- Our selector tool helps you to find the most relevant journal

- We provide round the clock customer support

- Convenient online submission

- Thorough peer review

- Inclusion in PubMed and all major indexing services

- Maximum visibility for your research

Submit your manuscript at www.biomedcentral.com/submit
) Biomed Central 